\title{
Plasma Enhanced Atomic Layer-etched and Regrown GaN-on-GaN High Power $p$-n Diodes
}

Prudhvi Ram Peri, Kevin Hatch, Daniel Messina, Kai Fu, Yuji Zhao, Robert Nemanich and David Smith Arizona State University, Tempe, Arizona, United States

Gallium nitride has widespread applications in the fields of optoelectronics, photonics and electronics due to its attractive physical and electrical properties such as larger band gap, higher critical electric field and switching frequency, which lead to its use in the fabrication of high-voltage devices with efficient power conversion and high-power utilization [1]. Freestanding GaN substrates grown by high vapor pressure epitaxy (HVPE) make it possible to grow devices with reduced defect density $\left(<10^{-4} \mathrm{~cm}^{-2}\right)$, unlike GaN devices grown on foreign substrates [2]. The etching of mesa structures is commonly used to terminate or isolate high-voltage $\mathrm{GaN}$ devices with complex device structures in order to reduce low-voltage breakdown at junction edges. Unlike etch termination techniques for Si semiconductors, suitable schemes for $\mathrm{GaN}$ devices are still under development [3]. Although the density of threading defects (TD) is reduced in epitaxial devices, dislocations caused by etch damage are liable to increase the severity of breakdown and reduce the reliability of the devices. TDs are known to act as trap states in GaN films, and are considered as being responsible for local high reverse leakage currents due to the presence of metastable acceptor and donor like states in their vicinity [4]. The development of reliable in situ etching techniques should be beneficial in creating complicated device structures and selective-area doping with minimal etch damage. In this work, two devices (labeled here as A1 and A2) were grown homoepitaxially by metalorganic chemical vapor deposition on 2-inch c-plane $n+\mathrm{GaN}$ substrates with a carrier concentration of $\sim 10^{18} \mathrm{~cm}^{-2}$ at a growth temperature of $\sim 1040^{\circ} \mathrm{C}$ [5]. Approximately $4-5$ microns of unintentionally-doped (UID) GaN was grown on each device followed by plasma-enhanced atomic layer etching (PEALE) with a precise etch rate of $5 \mathrm{~A} /$ cycle. This precise etching was achieved by application of "supercycles" consisting of surface oxidation, by a remote RF oxygen plasma, followed by five alternating exposures of hydrogen fluoride and trimethylgallium (TMGa) for fluorination and ligand exchange to remove the surface oxide. The removal rate and thickness measurements were conducted using an in situ multiwavelength ellipsometer. Hydrogen-nitrogen plasma passivation was performed on device A2 to remove surface impurities and maintain $\mathrm{Ga} / \mathrm{N}$ ratio. $\mathrm{Mg}$-doped $p$-GaN layers with thicknesses in the range of 400$500 \mathrm{~nm}$ was then grown on all samples. Samples suitable for cross-sectional TEM observation were prepared using a dual-beam FEI NOVA 200 FIB system, with initial thinning at $30 \mathrm{keV}$ and final thinning at $5 \mathrm{keV}$. SEM images were taken on the same instrument. A Philips-FEI CM-200 FEG TEM operated at $200 \mathrm{keV}$ was used for imaging. The surface composition after etching for device A1 was determined by in situ X-ray photoelectron spectroscopy (XPS), as shown in Fig. 1(a), which reveals the presence of oxygen on the surface. Plan-view SEM images of the regrown surface show closely spaced GaN islands with circular growth interruptions of about $20 \mu \mathrm{m}$. The low magnification SEM image, Fig. 1(b), shows a uniform surface with growth interruptions in the form of dark spots measured to be $\sim 20 \mu \mathrm{m}$ in diameter. The dark spots correspond to interruptions in the $p$-GaN growth, as observed in Fig. 1(c). The GaN islands can be observed at higher magnification in Fig. 1(d). Figure 2 shows a series of cross-sectional TEM images of the device A1. The $p$-GaN and UID-GaN interface can be clearly observed in Fig. 2(a), and in the high-resolution image in Fig. 2(b). Threading dislocations and dislocation loops can be observed within the $p$-GaN in Figs. 2(c) and 2(d). The presence of defects in the $p$-GaN was not due to the quality of UID-GaN or substrate, since no defects were observed in them. This clearly indicates the presence of oxygen at the surface before the regrowth of $p-\mathrm{GaN}$ effected the device quality. The presence of such dislocations will reduce device performance drastically and cause premature breakdown [6]. 

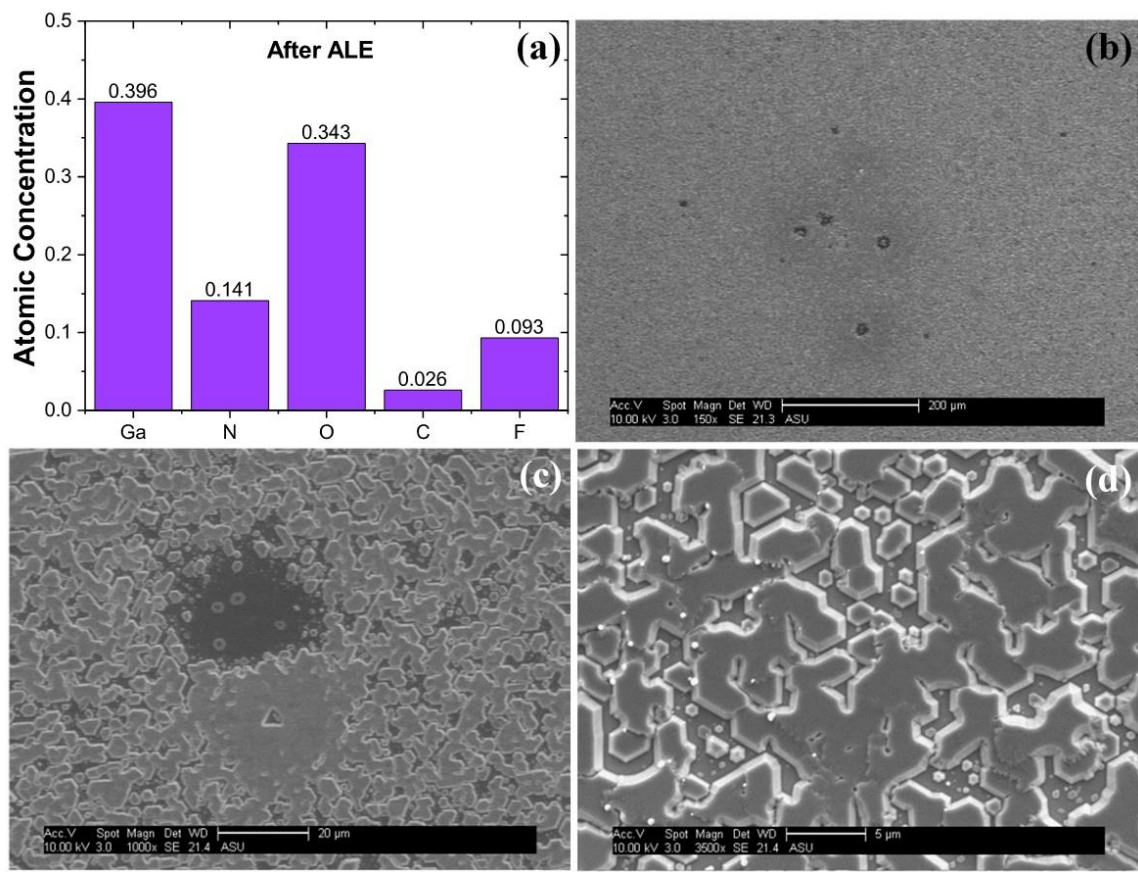

Figure 1. (a) Surface composition of device A1 before p-GaN regrowth and measured by XPS; (b) Low magnification plan-view SEM image shows uniform surface with growth interruptions; (c) Image focused on the growth interruption area with diameter of $\sim 20 \mu \mathrm{m}$; and (d) Image focused on uniform area shows GaN islands.
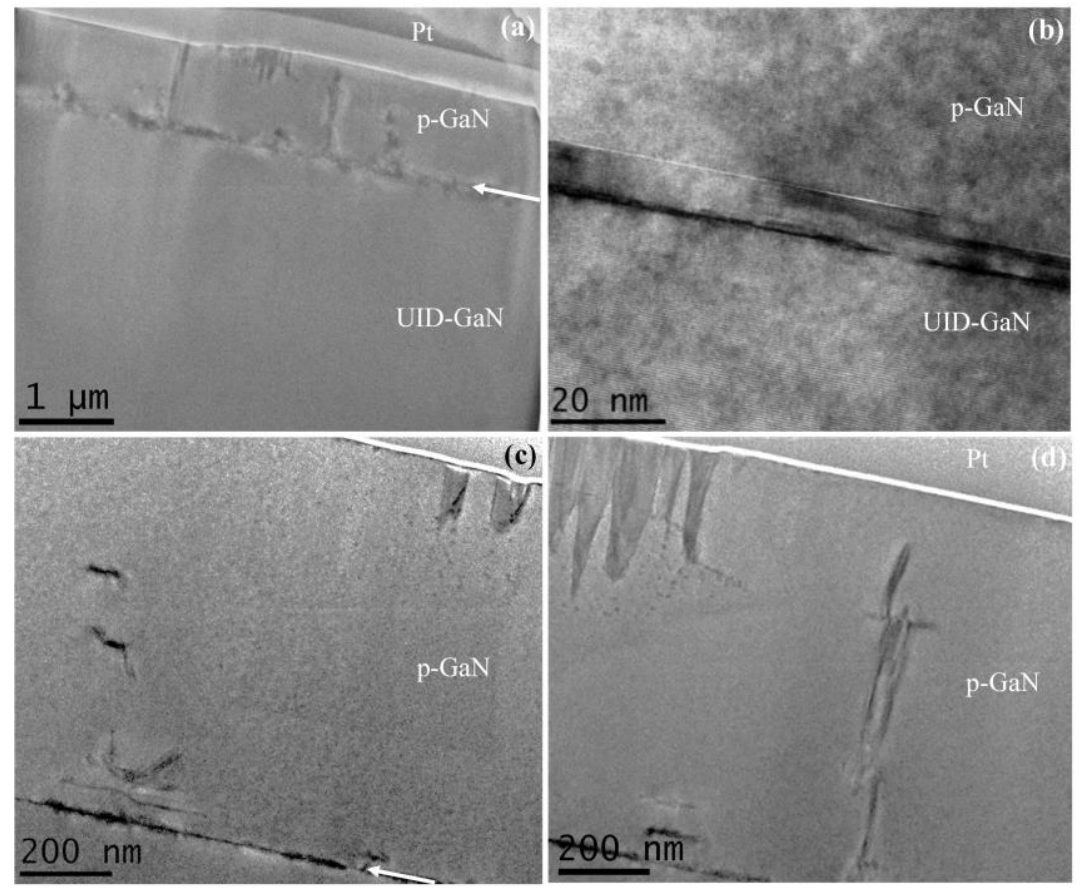

Figure 2. (a) Cross-sectional TEM image showing $p-G a N / U I D-G a N$ interface (white arrow) and dislocations within p-GaN; (b) TEM image of p-GaN/UID-GaN at high magnification showing basalplane stacking fault; (c) TEM image showing dislocation loops within p-GaN near the interface; and (d) TEM image showing defects originating at the interface and extending to the surface within p-GaN layer. 


\section{References}

1. Y. Zhao, H. Fu, G. T. Wang, and S. Nakamura, Adv. Opt. Photonics 10, 246 (2018).

2. I. C. Kizilyalli, A. P. Edwards, O. Aktas, T. Prunty, and D. Bour, IEEE Trans. Electron Devices 62, (2015).

3. T. Oka, Y. Ueno, T. Ina, and K. Hasegawa, (2014).

4. P. J. Hansen, et al., Appl. Phys. Lett 72, 2247 (1998).

5. J. K. Sheu, M. L. Lee, and W. C. Lai, Appl. Phys. Lett 86, 52103 (2005).

6. This work was supported by ARPA-E award DE-AR0000868. The authors acknowledge the use of facilities within the John M. Cowley Center for High Resolution Electron Microscopy at Arizona State University. 